\title{
Therapie als Teenager - Schutz auch noch als Twen
}

\author{
Die 2002 publizierten 3-Jahres-Daten der PAT-Studie zeigten, dass \\ eine subkutane spezifische Immuntherapie Kinder mit allergischer \\ Rhinitis vor Asthma schützen kann. Jetzt liegen die 5-Jahres-Daten \\ vor, die noch dieses Jahr in Allergy publiziert werden sollen. Erstautor \\ Prof. Dr. Bodo Niggemann erläutert vorab die wichtigsten Ergebnisse.
}

$?$

Nach den 3-Jahres-Daten der PAT-

Studie lässt sich das Auftreten von Asthma bei Kindern mit allergischer Rhinitis durch eine subkutane spezifische Immuntherapie mit einer Odds Ratio von 2,52 verhindern. Wie sah das Ergebnis nach 5 Jahren aus?

Niggemann: Die 5-Jahres-Daten sehen noch etwas besser aus - die Odds Ratio betrug 2,68. Zu diesem Punkt haben wir zudem bereits die 10-Jahres-Daten: Hier lag die Odds Ratio sogar bei 3,19. Mit anderen Worten: Auch 7 Jahre nach Beendigung der Hyposensibilisierung ist eine Asthmaprävention nachzuweisen.

Konnten auch die positiven Effekte auf die bronchiale Hyperreagibilität bestätigt werden?

Niggemann: Hier haben wir nach 5 Jahren - anders als nach 3 Jahren - sowohl während der Saison als auch im Winter nicht nur in der Verum-, sondern auch in der Kontrollgruppe einen Anstieg der Methacholin-Reizschwelle, d. h. einen Abfall der Hyperreagibilität gemessen. Der Effekt war jedoch in der Verumgrup-

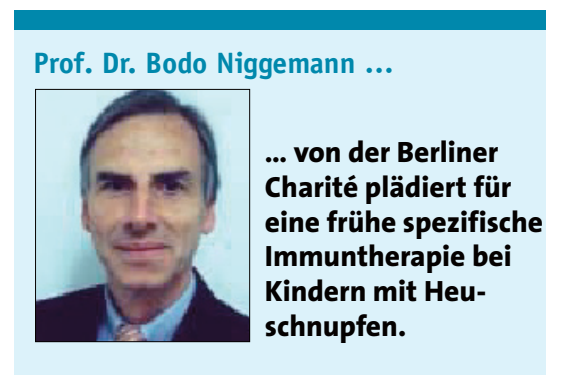

pe stärker ausgeprägt. Wir vermuten, dass die bronchiale Hyperreagibilität bei Kindern im natürlichen Verlauf mit zunehmendem Alter abnimmt, dieser Prozess jedoch durch die Hyposensibilisierung beschleunigt wird. Wir dachten zunächst, dies sei ein spezifischer Effekt, aber wahrscheinlich ist es nur ein zeitlicher.

Wie war die Wirksamkeit der Hypo-
sensibilisierung hinsichtlich der Symp-
tome der allergischen Rhinitis 2 Jahre nach
ihrer Beendigung?
Niggemann: Beim konjunktivalen Pro-
vokationstest beispielsweise war die Reiz-
schwelle für Gräser und Birkenpollen in
der Kontrollgruppe immer sehr niedrig.

\section{Die PAT-Studie auf einen Blick}

Da etwa $20 \%$ aller Patienten mit Heuschnupfen später Asthma entwickeln, sollte in der Studie Preventive Allergy Treatment (PAT) in den Jahren 1992-94 überprüft werden, ob die subkutane spezifische Immuntherapie (SCIT) diesen Etagenwechsel verhindern kann. Dazu wurden die Daten von 205 Kindern im Alter von 6-14 Jahren mit allergischen Rhinitis dokumentiert, die gegen Gräser und/oder Birkenpollen sensibilisiert waren. Die Kinder wurden einer Verum- oder einer Kontrollgruppe zugeordnet. Beide Gruppen erhielten eine symptomatische Therapie, in der Verumgruppe erfolgte darüber hinaus eine 3-jährige SCIT.

Vor Beginn der SCIT hatten $20 \%$ der Kinder milde Asthmasymptome während der Saison. Bei den übrigen $80 \%$ der Kinder traten innerhalb von 3 Jahren in der SCIT-Gruppe signifikant seltener Asthmasymptome auf als in der Kontrollgruppe: Die Odds Ratio lag bei 2,52 $(p<0,05)$. Zudem ließ sich die bronchiale Hyperreagibilität in der SCIT-Gruppe während und außerhalb der Saison signifikant reduzieren ( $p<0,05$ bzw. 0,001), in der Kontrollgruppe besserte sie sich hingegen nicht.

Möller C et al. Pollen immunotherapy reduces the development of asthma in children with seasonal rhinoconjunctivitis (the PAT-study). J Allergy Clin Immunol 2002; 109: 251-6
In der Verumgruppe stieg sie hingegen bis zum 3. Jahr ganz klar an. Nach 5 Jahren fiel sie wieder leicht ab, aber trotzdem war sie nach wie vor signifikant höher als in der Kontrollgruppe.

$\int$ Sint Sind bei der aktuellen Auswertung Überraschungen aufgetreten - beispielsweise unerwartete Nebenwirkungen? Niggemann: Nein, an der guten Verträglichkeit hat sich nichts geändert. Das einzig Unerwartete war die Besserung der bronchialen Hyperreagibilität in der Kontrollgruppe.

Welcher Mechanismus könnte dem präventiven Effekt zu Grunde liegen? Niggemann: Nach dem Konzept der "united airways" gehen wir davon aus, dass die Schleimhaut in der Nase sozusagen die Verlängerung der Schleimhaut in der Lunge darstellt. Zudem sehen wir die Allergie als eine Systemerkrankung an, die sich an verschiedenen Orten manifestiert. Und da die subkutane Hyposensibilisierung eine systemische Therapie ist, kann sie auch an verschiedenen Orten wirken.

Welche Kinder mit allergischer Rhinitis sollten eine spezifische Immuntherapie erhalten?

Niggemann: Viele! Voraussetzung ist, dass es sich um eine IgE-vermittelte allergische Erkrankung handelt, deren Symptome in einer zweiten Saison reproduziert werden können. Zudem müssen die klinische Relevanz des Allergens gesichert, die Exposition nicht vermeidbar und das Allergenspektrum eher eng sein. Außerdem sollten die Kinder in der Regel älter als 6 Jahre sein, weil sie die Therapie dann locker mitmachen. Die Schwere der Symptome spielt eher eine untergeordnete Rolle - wenn ein Patient mit leichten Symptomen einen hohen Leidensdruck hat oder den asthmapräventiven Effekt wünscht, würden wir die Hyposensibilisierung empfehlen. Nur bei sehr schweren Symptomen ist sie weniger empfehlenswert, weil man dann oft aufgrund von unerwünschten Reaktionen die notwendige Erhaltungsdosis nicht erreicht.

Herr Prof. Niggemann, vielen Dank für - das Gespräch.

Das Interview führte Petra Eiden, Berlin. 